\title{
Structural Stability of Functionalized Silicene Nanoribbons with Normal, Reconstructed, and Hybrid Edges
}

\author{
Sadegh Mehdi Aghaei, ${ }^{1}$ Ingrid Torres, ${ }^{1}$ and Irene Calizo ${ }^{1,2}$ \\ ${ }^{1}$ Department of Electrical and Computer Engineering, Florida International University, Miami, FL 33174, USA \\ ${ }^{2}$ Department of Mechanical and Materials Engineering, Florida International University, Miami, FL 33174, USA \\ Correspondence should be addressed to Irene Calizo; calizo@gmail.com
}

Received 21 March 2016; Accepted 28 August 2016

Academic Editor: Yasuhiko Hayashi

Copyright (c) 2016 Sadegh Mehdi Aghaei et al. This is an open access article distributed under the Creative Commons Attribution License, which permits unrestricted use, distribution, and reproduction in any medium, provided the original work is properly cited.

Silicene, a novel graphene-like material, has attracted a significant attention because of its potential applications for nanoelectronics. In this paper, we have theoretically investigated the structural stability of edge-hydrogenated and edge-fluorinated silicene nanoribbons (SiNRs) via first-principles calculations. Various edge forms of SiNRs including armchair edge, zigzag edge, Klein edge, reconstructed Klein edge, reconstructed pentagon-heptagon edge, and hybrid edges have been considered. It has been found that fully fluorinated Klein edge SiNRs, in which each edge Si atom is terminated by three fluorine atoms, are the most stable structure. We also discovered that a hybrid edge structure of trihydrogenated Klein edge and dihydrogenated zigzag edge can increase the nanoribbon's stability up to that of dihydrogenated armchair edge SiNR, which is known as the most stable edgehydrogenated structure. With the attractive properties of silicene for practical applications, the obtained results will advance experimental investigations toward the development of silicene based devices.

\section{Introduction}

Since its discovery [1, 2], graphene has been found to be an attractive material owing to its wide range of applications [3-9]. Nonetheless, fabrication of graphene-based devices has been impeded by difficulties in synthesizing large area of graphene sheets, toxicity, and incompatibility with silicon-based CMOS. Silicon resembles carbon as they appear next to each other in the same group of periodic table. Recently silicene, the silicon equivalent of graphene, has captured tremendous attention since it offers similar benefits as graphene but with fewer challenges [10-14]. Numerous outstanding properties such as ferromagnetism [15], half-metallicity [16], quantum Hall effect [17], and superconductivity [18] have been reported. Silicene was first theoretically studied by Takeda and Shiraishi in 1994 [19] and then reconsidered by GuzmánVerri and Lew Yan Voon in 2007 [20], calling it silicene. Silicene has been experimentally grown on many substrates such as $\mathrm{Ag}$ [21-23], $\operatorname{Ir}$ [24], $\mathrm{ZrB}_{2}$ [25], and $\mathrm{ZrC}$ [26]. It possesses electronic properties similar to graphene; for example, no band gap has been observed in silicene. The Dirac cones are also seen in silicene because of the linear crossing of the conduction and valence band at the Fermi level $[27,28]$. As a result, the electron acts like massless fermions and moves through silicene with a high Fermi velocity of $10^{5}-10^{6} \mathrm{~ms}^{-1}$ [29]. Unlike a flat graphene sheet, the stable silicene sheet is buckled [30,31], due to the tendency of silicon atoms to accept $\mathrm{sp}^{2}$ and $\mathrm{sp}^{3}$ hybridization rather than $\mathrm{sp}^{2}$ hybridization. The extraordinary properties of silicene along with its compatibility with silicon-based nanoelectronics give an edge to silicene over graphene in this respect. Numerous potential applications of silicene in spintronics [32], FETs [33-35], and sensing devices [36, 37] have been proposed.

One technique used to open a band gap in silicene is through silicene nanoribbons (SiNRs) [19]. SiNRs were synthesized on $\mathrm{Ag}(110)$ and $\mathrm{Au}$ (110) substrates [38-41]. It is predicted that nanoribbons can also be fabricated using nanolithography techniques [42-44]. Like graphene [45], armchair and zigzag/Klein edges are obtained by cutting 
the silicene honeycomb lattice along $\langle 1 \overline{1} 10\rangle$ and $\langle 2 \overline{1} \overline{1} 0\rangle$ directions, respectively. The chiral edges are the intermediate orientations which consist of hybrid edge types. Numerous studies have been performed to explore the outstanding properties of SiNRs [46-55].

Nonetheless, so far, no in depth study has investigated the full range of SiNRs edge types. In this paper, firstprinciples method based on density functional theory (DFT) was employed to investigate the effects of edge hydrogenation $(-\mathrm{H})$ and fluorination $(-\mathrm{F})$ on the stability of different SiNRs edge types. Our results will help experimental and theoretical investigations continue to advance Si-based technology.

The paper is organized as follows. Section 2 describes the details of our first-principles calculations used to investigate the stability of SiNRs, results and discussions are detailed in Section 3, and we present our conclusions in Section 4.

\section{Computational Methods}

First-principles calculations based on DFT method are employed to perform the calculation using Atomistix ToolKit (ATK) software package [56-58]. The Perdew Burke Ernzerhof (PBE) parameterized Generalized Gradient Approximation (GGA) exchange correlation with a double- $\zeta$ polarized basis set with a mesh cut-off energy of 150 Rydberg is utilized. A vacuum space of $15 \AA$ is employed on each side of the supercell to suppress mirroring interaction. Also, the electronic temperature was set to $300^{\circ} \mathrm{K}$. All the atomic positions and lattice parameters are optimized with the maximum force and stress of $0.05 \mathrm{eV} / \AA$ and $0.001 \mathrm{eV} / \AA^{3}$, respectively. During optimization, a $k$ mesh of $1 \times 1 \times 11$ is used on our $1 D$ structure. The $k$ mesh is later increased to $1 \times 1 \times 121$ to gather accurate results.

\section{Results and Discussions}

Similar to graphene [45], armchair and zigzag/Klein edges can be made from a silicene sheet by cutting along the $\langle 1 \overline{1} 10\rangle$ and $\langle 2 \overline{1} \overline{1} 0\rangle$ directions, respectively, which are called normal edges (Figure 1). Besides these edges, SiNRs with Klein edge can be reconstructed to enhance the nanoribbons' stability. As a result, a reconstructed Klein edge and reconstructed pentagon-heptagon edge will be formed. To differentiate edge structures we categorized them, similar to [59], as follows: $a$ : armchair edge, $z$ : zigzag edge, $k$ : Klein edge, $r k$ : reconstructed Klein edge, and (5-7): reconstructed pentagon-heptagon edge. Moreover, we added subscripts which show the number of functional atoms; for example, $z_{2}$ indicates that all the dangling edge $\mathrm{Si}$ atoms are terminated by two functional atoms in the supercell. In order to separate the number of functional atoms of the two edges, we added a hyphen to the subscripts; for example, $z_{2-1}$ shows that the edge Si atoms of one edge are terminated by two functional atoms, while on the other edge, they are attached to one functional atom in the supercell. In this paper, we have theoretically examined the stability of edge functionalized armchair, zigzag, Klein, reconstructed Klein, and hybrid edges. To investigate the effects of edge functionalization on the stability of SiNRs, $X$
TABLE 1: Edge formation energy $E_{\text {edge }}(\mathrm{eV})$ of different configurations of functionalized edge ASiNRs.

\begin{tabular}{lcc}
\hline Edge type & Edge atom & $E_{\text {edge }}(\mathrm{eV} / \AA ̊ \Omega$ \\
$a_{11}$ & Bare & +0.33 \\
& $\mathrm{H}$ & -0.08 \\
& $\mathrm{~F}$ & -1.03 \\
\hline \multirow{2}{*}{$a_{22}$} & Bare & +0.33 \\
& $\mathrm{H}$ & -0.12 \\
& $\mathrm{~F}$ & -2.48 \\
\hline \multirow{3}{*}{$a_{22-11}$} & Bare & +0.33 \\
& $\mathrm{H}$ & -0.02 \\
& $\mathrm{~F}$ & -1.75 \\
\hline \multirow{2}{*}{$a_{21}$} & Bare & +0.33 \\
& $\mathrm{H}$ & +0.05 \\
& $\mathrm{~F}$ & -1.69 \\
\hline
\end{tabular}

atoms $(X=\mathrm{H}$ and $\mathrm{F})$ with different densities on the edges are considered.

3.1. Armchair Edge Silicene Nanoribbons. Figure 2 shows different configurations of functionalized edge ASiNRs including $a_{11}$ (mono- $\mathrm{H}$ or $\mathrm{F}$ atom on the both edges), $a_{22}$ (di-H or -F on the both edges), $a_{22-11}$ (di-H or -F on the one edge and mono- $\mathrm{H}$ or $-\mathrm{F}$ on the other edge), and $a_{21}$ (both edges are periodically terminated by di- $\mathrm{H}$ or $-\mathrm{F}$ and mono- $\mathrm{H}$ or $-\mathrm{F}$ ).

In order to measure the stability of functionalized edge SiNRs, we calculated edge formation energy which is described as

$$
E_{\text {edge }}=\frac{\left(E_{\text {ribbon }}-n_{\mathrm{Si}} \times E_{\mathrm{Si}}-\left(n_{X} / 2\right) \times E_{X_{2}}\right)}{2 L} .
$$

Here, $E_{\text {ribbon }}$ stands for the total energy of the functionalized edge SiNR. $X$ is the functional addend which can be $\mathrm{H}$ or $\mathrm{F}$ atom. $E_{\mathrm{Si}_{1}}$ and $E_{X_{2}}$ represent, respectively, the total energy of free $\mathrm{Si}$ and isolated hydrogen or fluorine molecule. The numbers of $\mathrm{Si}$ atoms and functional addends per supercell are expressed by $n_{\mathrm{Si}}$ and $n_{X}$, respectively. $L$ describes the nanoribbons' periodic length and is multiplied by two because of the two nanoribbons' edge. Under perfect vacuum condition, the negative edge formation energy shows that the chemical reaction is exothermic. Table 1 presents the edge formation energies for different configuration of functionalized edge ASiNRs. As we expected, the nanoribbon with bare edges is unstable with $E_{\text {edge }}$ of $+0.33 \mathrm{eV} / \AA$. Edge functionalization improves the structure's stabilities. $a_{22}$ edged nanoribbons have the most negative edge formation energies for both the edge-hydrogenated $(-0.12 \mathrm{eV} / \AA)$ and edge-fluorinated $(-2.48 \mathrm{eV} / \AA \AA)$ cases. It can be also understood that fluorination of SiNRs' edges makes the SiNRs much more stable than hydrogenation.

In order to investigate the effects of experimental conditions on the stability of SiNRs, we consider the gas pressure and temperature of the process in our calculations. To this end, we have compared the calculated edge formation energy 


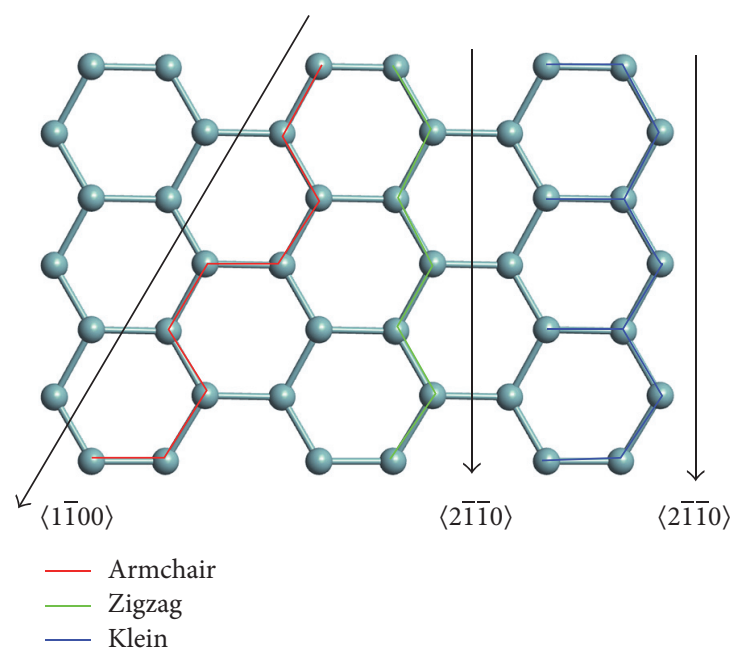

Figure 1: Depending on the cutting directions $(\langle 1 \overline{1} 10\rangle$ and $\langle 2 \overline{1} \overline{1} 0\rangle)$, armchair and parallel zigzag/Klein edges can be formed by cutting silicene honeycomb sheet.
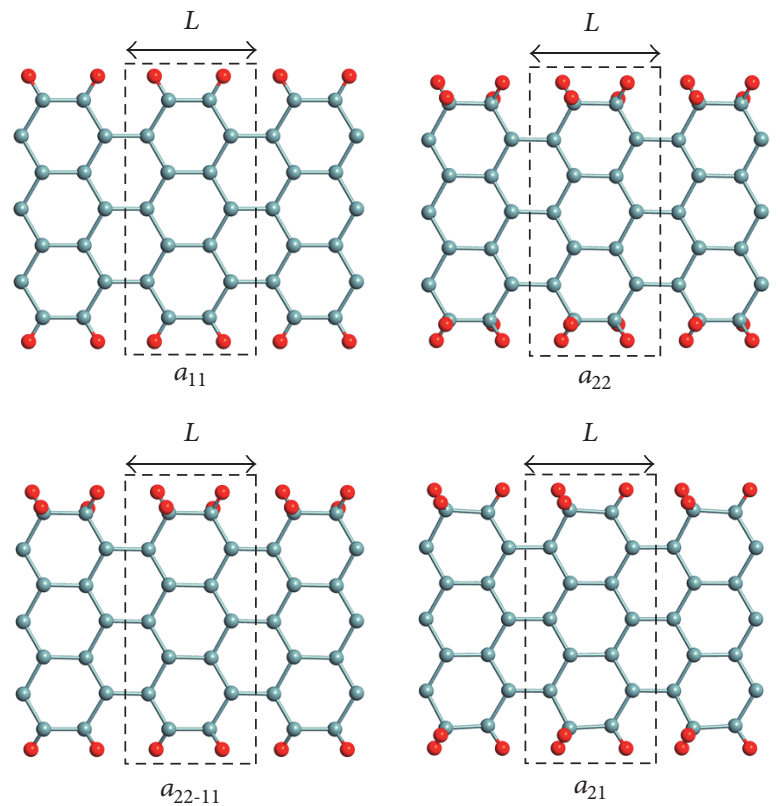

Figure 2: Various configurations of functionalized edge ASiNRs. The cyan and red balls represent Si and functional addends (H or F), respectively. The black dashed box shows the primitive supercell. $L$ is the length of the supercell.

to the hydrogen or fluorine molecule's chemical potential $\left(\mu_{X_{2}}\right)$ expressed as

$$
G_{\text {Func }}=E_{\text {edge }}-\frac{1}{2} \times \rho_{X} \times \mu_{X_{2}} .
$$

Here, $G_{\text {Func }}$ is the Gibbs free energy. $\rho_{X}$ is the edge density of functional addends which is equal to $n_{X} / 2 L$. The temperature and gas pressure dependence $\left(\mu_{X_{2}}\right)$ is described by

$$
\mu_{X_{2}}=H^{o}(T)-H^{o}(0)-T S^{o}(T)+k_{B} T \ln \left(\frac{P}{P^{o}}\right),
$$

where $H^{o}\left(S^{o}\right)$ is the enthalpy (entropy) of functional addends at the pressure $P^{o}=1$ bar and can be calculated using [60].
Figure 3(a) shows the values of the Gibbs free energy of $\mathrm{H}_{2}$ molecule versus $\mathrm{H}_{2}$ molecule's chemical potential for different configurations of functionalized edge ASiNRs. When $\mu_{\mathrm{H}_{2}}$ is less than $-1.4 \mathrm{eV}, a_{11}$ is the most stable structure. Whereas, once $\mu_{\mathrm{H}_{2}}$ is greater than $-1.4 \mathrm{eV}, a_{22}$ becomes the most stable one. Furthermore, under ambient conditions $(T=300 \mathrm{~K}$, $P=5 \times 10^{-7}$ bar, and $\left.\mu_{\mathrm{H}_{2}}=-0.7 \mathrm{eV}\right), a_{22}$ has the most negative value of energy. For fluorinated edge ASiNRs (see Figure $3(\mathrm{~b})), a_{22}$ is found as the most stable structure for the whole range of chemical potentials.

3.2. Zigzag, Klein, Reconstructed, and Hybrid Edge Silicene Nanoribbons. As shown in Figure 1, cutting a silicene sheet 


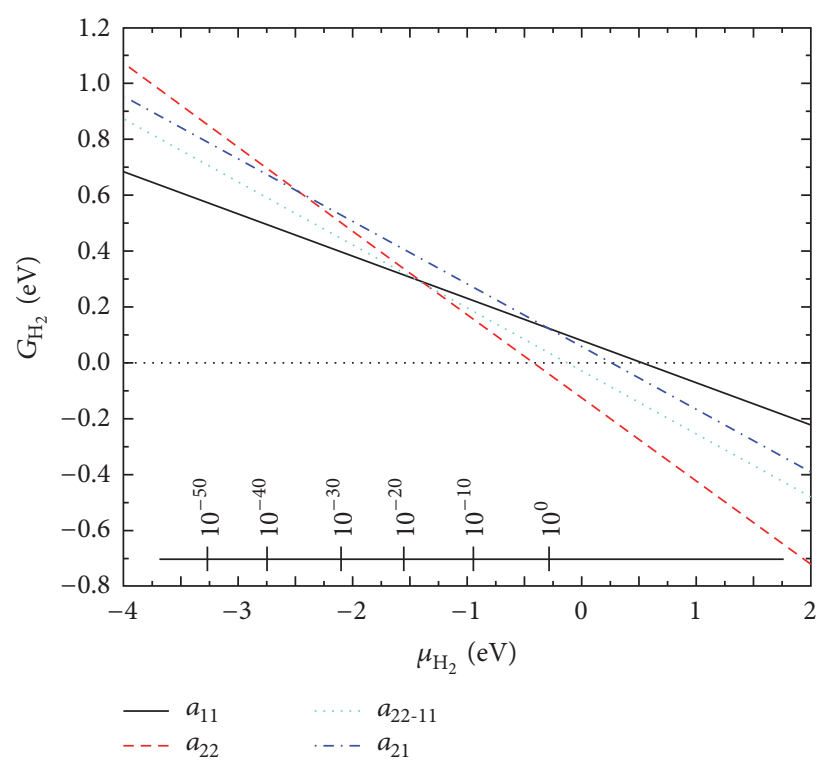

(a)

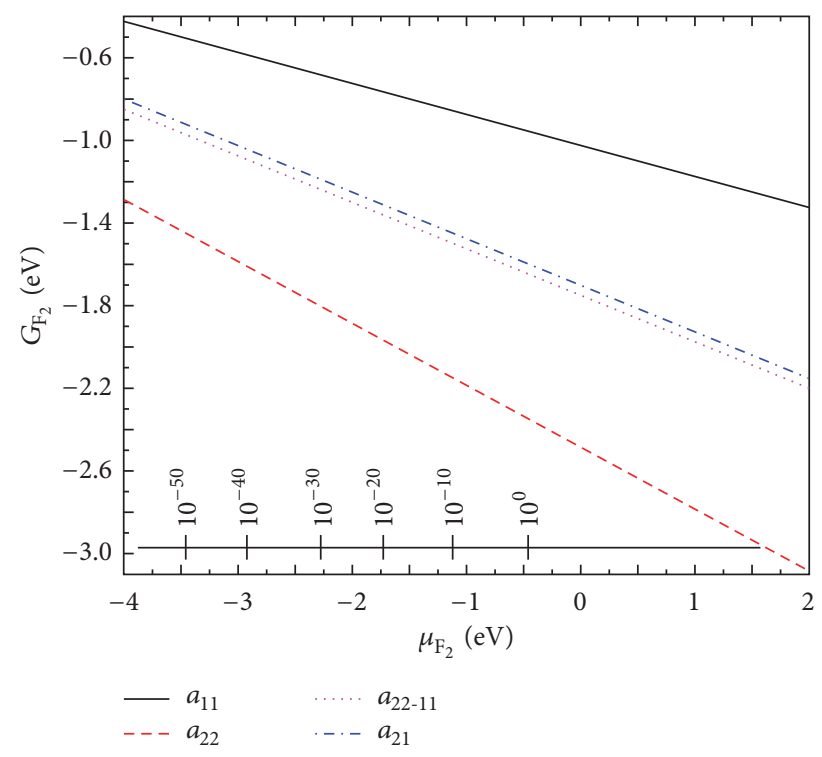

(b)

FIGURE 3: Gibbs free energy of (a) hydrogenated and (b) fluorinated edge ASiNRs as a function of chemical potential. The bottom inset axis represents the pressure (bar) of (a) molecular $\mathrm{H}_{2}$ corresponding to $\mu_{\mathrm{H}_{2}}$ and (b) molecular $\mathrm{F}_{2}$ corresponding to $\mu_{\mathrm{F}_{2}}$ when $T=300 \mathrm{~K}$.
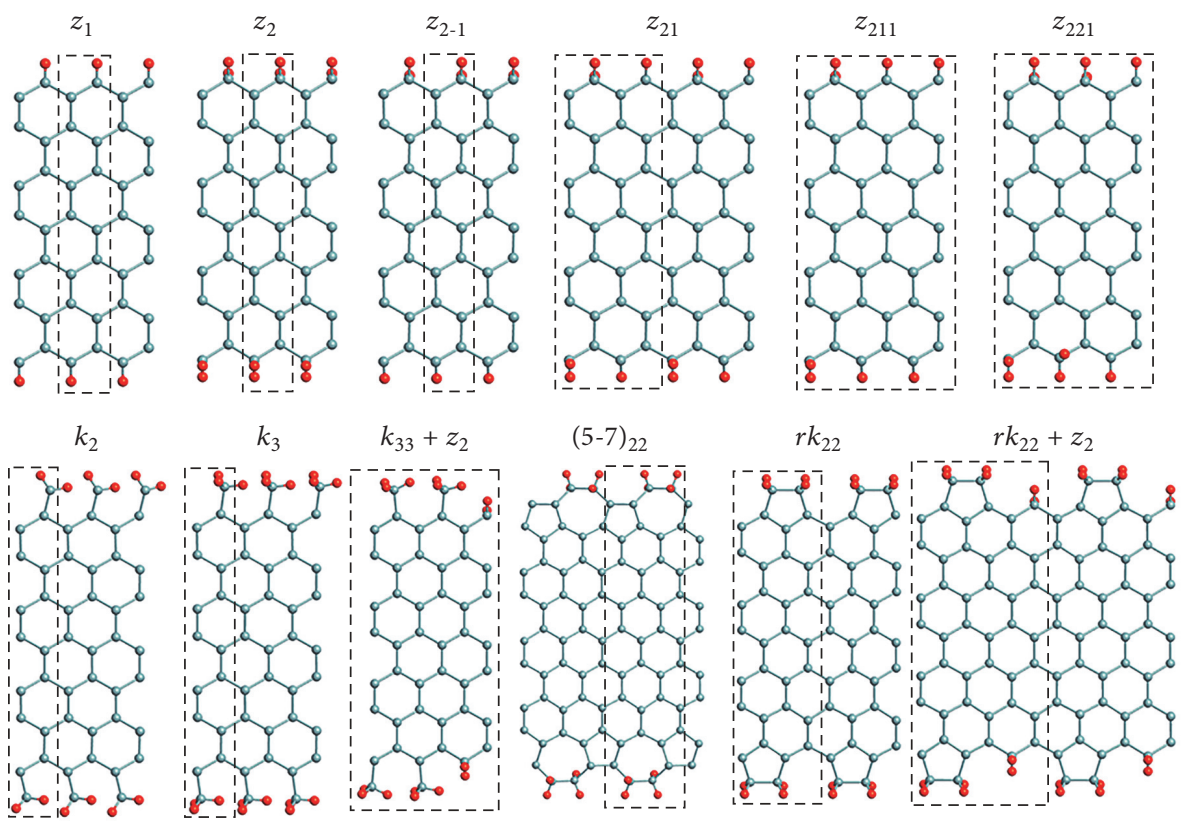

Figure 4: Various edge functionalized SiNRs along $\langle 2 \overline{1} \overline{1} 0\rangle$ direction. The cyan and red balls represent Si and functional addends (H or F), respectively. The dashed boxes show the primitive supercells.

along the $\langle 2 \overline{1} \overline{1} 0\rangle$ direction makes it possible to have two parallel zigzag and Klein edges. As a result, the $\langle 2 \overline{1} \overline{1} 0\rangle$ orientation is expected to be more complex than $\langle 1 \overline{1} 10\rangle$. Different configurations of functionalized edge SiNRs along $\langle 2 \overline{1} \overline{1} 0\rangle$ direction including normal edge (zigzag and Klein), reconstructed edge (reconstructed Klein and reconstructed pentagon-heptagon), and hybrid edge structures (mixture of edges) are evaluated in this study, as shown in Figure 4. The edge formation energies of $z_{1}, z_{2}, z_{2-1}, z_{21}, z_{211}$, and $z_{221}$ are listed in Table 2. Similar to ASiNRs, the bare ZSiNRs are unstable. Through edge functionalization, the stabilities of the ZSiNRs are increased. The fully hydrogenated ZSiNR $\left(z_{2}\right)$ with edge formation energy of $-0.01 \mathrm{eV} / \AA$ is found as the most stable ZSiNR. Since Si atoms tend to accept $\mathrm{sp}^{3}$ hybridizations over $\mathrm{sp}^{2}$ in silicene, the edge formation energy increases by full functionalization of Si edge atoms. Therefore, 


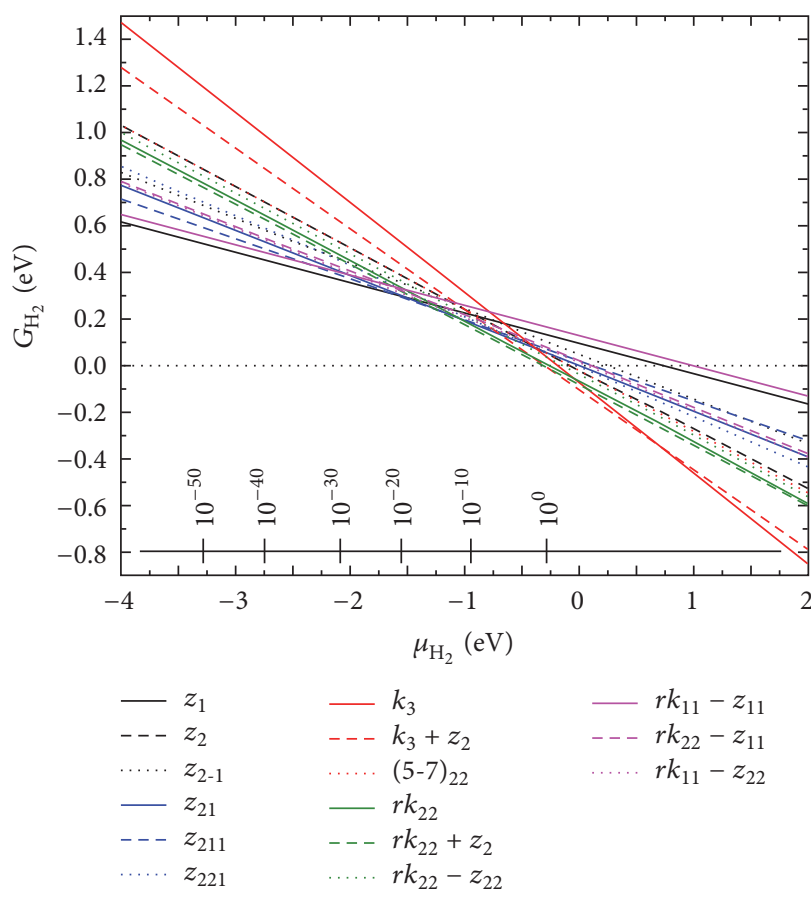

(a)

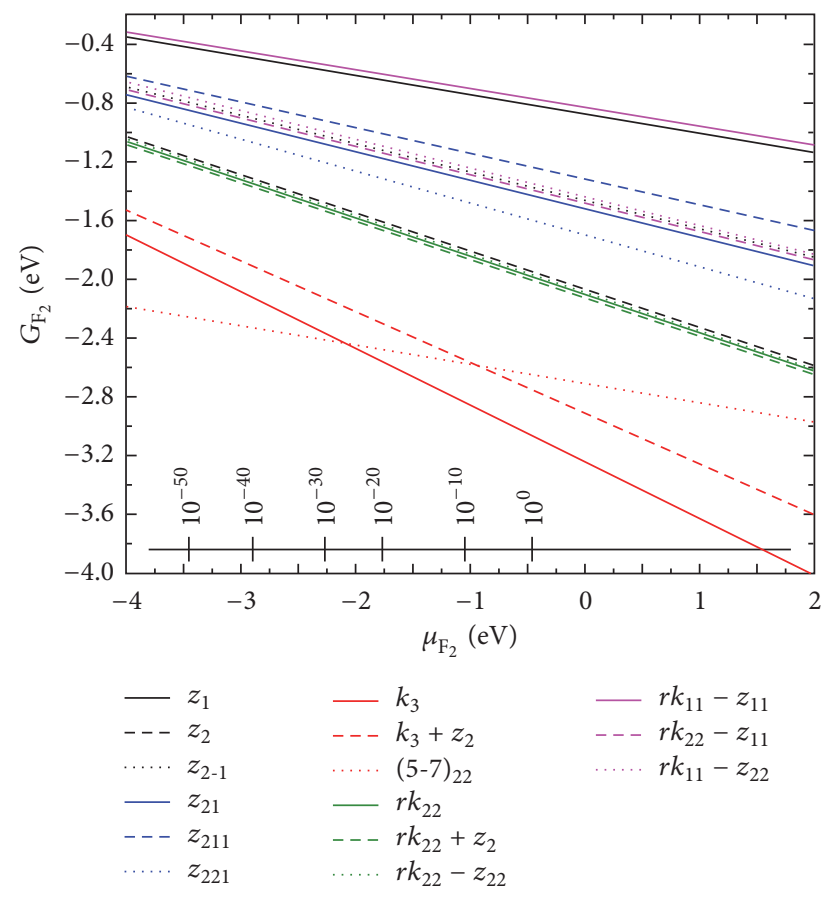

(b)

FiguRE 5: Gibbs free energy of different edge structures of (a) hydrogenated and (b) fluorinated SiNRs along $\langle 2 \overline{1} \overline{1} 0\rangle$ versus chemical potential. The bottom inset axis represents the pressure (bar) of (a) molecular $\mathrm{H}_{2}$ corresponding to $\mu_{\mathrm{H}_{2}}$ and (b) molecular $\mathrm{F}_{2}$ corresponding to $\mu_{\mathrm{F}_{2}}$ when $T=300 \mathrm{~K}$.

the monohydrogenated ZSiNR $\left(z_{1}\right)$ has the lowest stability among the ZSiNRs. In a similar manner to ASiNRs, fluorinating would raise the stability of the ZSiNRs $(-2.06 \mathrm{eV} / \AA$ ).

The positive value of edge formation energy for bare Klein edge SiNR $(+0.38 \mathrm{eV} / \AA)$ confirms that it is unstable. It is discovered that even dihydrogenating of SiNRs' Klein edge keep them unstable $(+0.25 \mathrm{eV} / \AA)$. However, trihydrogenated $k_{3}$ is fairly stable $(-0.72 \mathrm{eV} / \AA)$ due to the $\mathrm{sp}^{3}$ hybridization of Si edge atoms. On the other hand, fluorination will result in stable $k_{2}(-1.85 \mathrm{eV} / \AA)$ and high stable $k_{3}(-3.24 \mathrm{eV} / \AA)$ SiNRs. Therefore, the trifluorinated Klein edge SiNR is the most stable structure among all the edge structures (see Table 2). We have also studied different forms of Klein/zigzag hybrid SiNRs. It is found that the edge formation energy of hydrogenated $k_{33}+z_{2}$ (periodic Klein vacancies which are fully functionalized by two $\mathrm{H}$ atoms) is $-0.10 \mathrm{eV} / \AA$, very close to that of hydrogenated $a_{22}(-0.12 \mathrm{eV} / \AA)$. As previously mentioned, reconstruction of bare Klein edges is possible due to its high instability. Our calculations show that bare reconstructed Klein edge ( $r k$ ) and reconstructed pentagonheptagon zigzag edge (5-7) are a slightly more stable than that of bare Klein edge (See Table 2). The monohydrogenated $r k$ and (5-7) are still unstable; however, full hydrogenation of edges makes them stable. Interestingly, the edge formation energies for mono- and difluorinated $r k$ and (5-7) are all negative, showing that they are all stable. The values of edge formation energies for fluorinated (5-7) $)_{22}$ and $r k_{22}$ are $-2.07 \mathrm{eV} / \AA$ and $-2.10 \mathrm{eV} / \AA$, respectively. The stability of $r k_{22}$ can be increased by periodically inserting a Klein edge vacancy and terminating it by two functional addends, making $r k_{22}+z_{2}$ structure. The hydrogenated (fluorinated) $r k_{22}+z_{2}$ is $0.01 \mathrm{eV} / \AA \AA(0.016)$ more stable than $r k_{22}$ (see Table 2). In conclusion, the fluorinated $k_{3}$ and hydrogenated $k_{33}+z_{2}$ were found to be the most stable structures along the $\langle 2 \overline{1} \overline{1} 0\rangle$ direction under perfect vacuum conditions.

In consideration of experimental conditions, the Gibbs free energies of the hydrogenated edge structures along the $\langle 2 \overline{1} \overline{1} 0\rangle$ direction were calculated using (2) and (3) (Figure 5(a)). For $\mu_{\mathrm{H}_{2}}$ is less than $-1.5 \mathrm{eV}, z_{1}$ has the most negative energy. When $-1.5 \mathrm{eV} \leq \mu_{\mathrm{H}_{2}}<-0.2 \mathrm{eV}, r k_{22}+z_{2}$ is the most stable structure. For $-0.2 \mathrm{eV} \leq \mu_{\mathrm{H}_{2}}<+0.75 \mathrm{eV}$, $k_{3}$ and $k_{33}+z_{2}$ have same Gibbs free energy. And finally, when $\mu_{\mathrm{H}_{2}} \geq+0.75 \mathrm{eV}, k_{3}$ is established as the most stable edge structure. Instead for fluorine, for $\mu_{\mathrm{F}_{2}}<-2 \mathrm{eV}$, (5-7) 22 structure and, for $\mu_{\mathrm{F}_{2}} \geq-1.9 \mathrm{eV}, k_{3}$ are found as the most stable structures (see Figure 5(b)). Under ambient conditions, that is, $300 \mathrm{~K}$ and $5 \times 10^{-7}$ bar, the hydrogenated $r k_{22}+z_{2}$ $\left(\mu_{\mathrm{H}_{2}}=-0.7 \mathrm{eV}\right)$ and fluorinated $k_{3}\left(\mu_{\mathrm{F}_{2}}=-0.9 \mathrm{eV}\right)$ are the most stable edges.

\section{Conclusion}

In summary, first-principles simulations based on DFT have been performed to study the stability of various configurations of edge-hydrogenated and fluorinated SiNRs. All possible edge types such as armchair, zigzag, Klein, reconstructed Klein, reconstructed (5-7), and hybrid edges are 
TABLE 2: Edge formation energy $E_{\text {edge }}(\mathrm{eV})$ of hydrogenated and fluorinated SiNRs along $\langle 2 \overline{1} \overline{1} 0\rangle$.

\begin{tabular}{|c|c|c|}
\hline Edge type & Edge atom & $E_{\text {edge }}(\mathrm{eV} / \AA)$ \\
\hline \multirow{3}{*}{$z_{1}$} & Bare & +0.38 \\
\hline & $\mathrm{H}$ & +0.097 \\
\hline & $\mathrm{F}$ & -0.87 \\
\hline \multirow{3}{*}{$z_{2}$} & Bare & +0.38 \\
\hline & $\mathrm{H}$ & -0.01 \\
\hline & $\mathrm{F}$ & -2.06 \\
\hline \multirow{3}{*}{$z_{2-1}$} & Bare & +0.38 \\
\hline & $\mathrm{H}$ & +0.05 \\
\hline & $\mathrm{F}$ & -1.46 \\
\hline \multirow{3}{*}{$z_{21}$} & Bare & +0.37 \\
\hline & $\mathrm{H}$ & +0.001 \\
\hline & $\mathrm{F}$ & -1.51 \\
\hline \multirow{3}{*}{$z_{211}$} & Bare & +0.35 \\
\hline & $\mathrm{H}$ & +0.024 \\
\hline & $\mathrm{F}$ & -1.31 \\
\hline \multirow{3}{*}{$z_{221}$} & Bare & +0.38 \\
\hline & $\mathrm{H}$ & -0.002 \\
\hline & $\mathrm{F}$ & -1.70 \\
\hline \multirow{3}{*}{$k_{2}$} & Bare & +0.38 \\
\hline & $\mathrm{H}$ & +0.25 \\
\hline & $\mathrm{F}$ & -1.85 \\
\hline \multirow{3}{*}{$k_{3}$} & Bare & +0.38 \\
\hline & $\mathrm{H}$ & -0.72 \\
\hline & $\mathrm{F}$ & -3.24 \\
\hline \multirow{3}{*}{$k_{33}+z_{2}$} & Bare & +0.33 \\
\hline & $\mathrm{H}$ & -0.10 \\
\hline & $\mathrm{F}$ & -2.90 \\
\hline \multirow{3}{*}{$(5-7)_{22}$} & Bare & +0.35 \\
\hline & $\mathrm{H}$ & -0.02 \\
\hline & $\mathrm{F}$ & -2.07 \\
\hline \multirow{3}{*}{$r k_{22}$} & Bare & +0.33 \\
\hline & $\mathrm{H}$ & -0.07 \\
\hline & $\mathrm{F}$ & -2.10 \\
\hline \multirow{3}{*}{$r k_{22}+z_{2}$} & Bare & +0.33 \\
\hline & $\mathrm{H}$ & -0.08 \\
\hline & $\mathrm{F}$ & -2.12 \\
\hline
\end{tabular}

studied. Results indicate that, by fluorination of SiNRs' edges, the stabilities tend to increase. Among all ASiNRs, $a_{22}$ has the highest chance of forming both experimentally under ambient conditions and theoretically under perfect vacuum conditions, no matter the functional addends. Along the $\langle 2 \overline{1} \overline{1} 0\rangle$ direction, finding the most stable structure is more complicated than that of $\langle 1 \overline{1} 10\rangle$. Functionalization of edge atoms by hydrogen makes $k_{33}+z_{2}$ and $r k_{22}+z_{2}$ the most stable structure under perfect vacuum and experimental ambient conditions, respectively. Finally, with fluorine as the functional addend, $k_{3}$ structure is formed under both experiment and vacuum conditions. It should be noted that other edge configurations can be obtained by changing the gas pressure and temperature under experimental conditions.

\section{Competing Interests}

The authors declare that there is no conflict of interests regarding the publication of this paper.

\section{Acknowledgments}

This work was supported in part by the Florida Education Fund's McKnight Junior Faculty Fellowship.

\section{References}

[1] K. S. Novoselov, A. K. Geim, S. V. Morozov et al., "Electric field effect in atomically thin carbon films," Science, vol. 306, no. 5696, pp. 666-669, 2004.

[2] K. S. Novoselov, A. K. Geim, S. V. Morozov et al., "Two-dimensional gas of massless Dirac fermions in graphene," Nature, vol. 438, no. 7065, pp. 197-200, 2005.

[3] S. Stankovich, D. A. Dikin, G. H. B. Dommett et al., "Graphenebased composite materials," Nature, vol. 442, no. 7100, pp. 282286, 2006.

[4] F. Schwierz, "Graphene transistors," Nature Nanotechnology, vol. 5, no. 7, pp. 487-496, 2010.

[5] X. Miao, S. Tongay, M. K. Petterson et al., "High efficiency graphene solar cells by chemical doping," Nano Letters, vol. 12, no. 6, pp. 2745-2750, 2012.

[6] W. J. Liu, L. Chen, P. Zhou et al., "Chemical-vapor-deposited graphene as charge storage layer in flash memory device," Journal of Nanomaterials, vol. 2016, Article ID 6751497, 6 pages, 2016.

[7] W. L. Zhang, J. Liu, and H. J. Choi, "Graphene and graphene oxide composites and their electrorheological applications," Journal of Nanomaterials, vol. 2015, Article ID 574637, 8 pages, 2015.

[8] X. Li, J. Shi, J. Pang, W. Liu, H. Liu, and X. Wang, "Graphene channel liquid container field effect transistor as $\mathrm{pH}$ sensor," Journal of Nanomaterials, vol. 2014, Article ID 547139, 6 pages, 2014.

[9] E. Akbari, R. Yusof, M. T. Ahmadi et al., "Bilayer graphene application on $\mathrm{NO}_{2}$ sensor modelling," Journal of Nanomaterials, vol. 2014, Article ID 534105, 7 pages, 2014.

[10] A. Kara, H. Enriquez, A. P. Seitsonen et al., "A review on silicene-new candidate for electronics," Surface Science Reports, vol. 67, no. 1, pp. 1-18, 2012.

[11] S. Z. Butler, S. M. Hollen, L. Cao et al., "Progress, challenges, and opportunities in two-dimensional materials beyond graphene," ACS Nano, vol. 7, no. 4, pp. 2898-2926, 2013.

[12] M. Xu, T. Liang, M. Shi, and H. Chen, "Graphene-like twodimensional materials," Chemical Reviews, vol. 113, no. 5, pp. 3766-3798, 2013.

[13] M. Houssa, A. Dimoulas, and A. Molle, "Silicene: a review of recent experimental and theoretical investigations," Journal of Physics Condensed Matter, vol. 27, no. 25, Article ID 253002, 2015.

[14] J. Zhuang, X. Xu, H. Feng, Z. Li, X. Wang, and Y. Du, "Honeycomb silicon: a review of silicene," Science Bulletin, vol. 60, no. 18, pp. 1551-1562, 2015. 
[15] X.-Q. Wang, H.-D. Li, and J.-T. Wang, "Induced ferromagnetism in one-side semihydrogenated silicene and germanene," Physical Chemistry Chemical Physics, vol. 14, no. 9, pp. 3031-3036, 2012.

[16] F.-B. Zheng and C.-W. Zhang, "The electronic and magnetic properties of functionalized silicene: a first-principles study," Nanoscale Research Letters, vol. 7, article 422, 2012.

[17] C.-C. Liu, W. Feng, and Y. Yao, "Quantum spin Hall effect in silicene and two-dimensional germanium," Physical Review Letters, vol. 107, no. 7, Article ID 076802, 2011.

[18] L. Chen, B. Feng, and K. Wu, "Observation of a possible superconducting gap in silicene on $\mathrm{Ag}(111)$ surface," Applied Physics Letters, vol. 102, no. 8, Article ID 081602, 2013.

[19] K. Takeda and K. Shiraishi, "Theoretical possibility of stage corrugation in $\mathrm{Si}$ and Ge analogs of graphite," Physical Review B, vol. 50, no. 20, pp. 14916-14922, 1994.

[20] G. G. Guzmán-Verri and L. C. Lew Yan Voon, "Electronic structure of silicon-based nanostructures," Physical Review B, vol. 76, no. 7, Article ID 075131, 2007.

[21] P. Vogt, P. De Padova, C. Quaresima et al., "Silicene: compelling experimental evidence for graphenelike two-dimensional silicon," Physical Review Letters, vol. 108, no. 15, Article ID 155501, 2012.

[22] B. Feng, Z. Ding, S. Meng et al., "Evidence of silicene in honeycomb structures of silicon on $\mathrm{Ag}(111)$," Nano Letters, vol. 12, no. 7, pp. 3507-3511, 2012.

[23] A. J. Mannix, B. Kiraly, B. L. Fisher, M. C. Hersam, and N. P. Guisinger, "Silicon growth at the two-dimensional limit on Ag(111)," ACS Nano, vol. 8, no. 7, pp. 7538-7547, 2014.

[24] L. Meng, Y. Wang, L. Zhang et al., "Buckled silicene formation on Ir(111)," Nano Letters, vol. 13, no. 2, pp. 685-690, 2013.

[25] A. Fleurence, R. Friedlein, T. Ozaki, H. Kawai, Y. Wang, and Y. Yamada-Takamura, "Experimental evidence for epitaxial silicene on diboride thin films," Physical Review Letters, vol. 108, no. 24, Article ID 245501, 2012.

[26] T. Aizawa, S. Suehara, and S. Otani, "Silicene on zirconium carbide (111)," The Journal of Physical Chemistry C, vol. 118, no. 40, pp. 23049-23057, 2014.

[27] S. Lebègue and O. Eriksson, "Electronic structure of twodimensional crystals from ab initio theory," Physical Review BCondensed Matter and Materials Physics, vol. 79, no. 11, Article ID 115409, 2009.

[28] H. Şahin, S. Cahangirov, M. Topsakal et al., "Monolayer honeycomb structures of group-IV elements and III-V binary compounds: first-principles calculations," Physical Review B, vol. 80, no. 15, Article ID 155453, 2009.

[29] L. Chen, C.-C. Liu, B. Feng et al., "Evidence for Dirac fermions in a honeycomb lattice based on silicon," Physical Review Letters, vol. 109, no. 5, Article ID 056804, 2012.

[30] S. Cahangirov, M. Topsakal, E. Aktürk, H. Şahin, and S. Ciraci, "Two- and one-dimensional honeycomb structures of silicon and germanium," Physical Review Letters, vol. 102, no. 23, Article ID 236804, 4 pages, 2009.

[31] M. Ezawa, "Valley-polarized metals and quantum anomalous hall effect in silicene," Physical Review Letters, vol. 109, no. 5, Article ID 055502, 2012.

[32] W.-F. Tsai, C.-Y. Huang, T.-R. Chang, H. Lin, H.-T. Jeng, and A. Bansil, "Gated silicene as a tunable source of nearly $100 \%$ spinpolarized electrons," Nature Communications, vol. 4, article 1500, 2013.
[33] H. Liu, J. Gao, and J. Zhao, "Silicene on substrates: a way to preserve or tune its electronic properties," Journal of Physical Chemistry C, vol. 117, no. 20, pp. 10353-10359, 2013.

[34] Z. Ni, H. Zhong, X. Jiang et al., "Tunable band gap and doping type in silicene by surface adsorption: towards tunneling transistors," Nanoscale, vol. 6, no. 13, pp. 7609-7618, 2014.

[35] L. Tao, E. Cinquanta, D. Chiappe et al., "Silicene field-effect transistors operating at room temperature," Nature Nanotechnology, vol. 10, no. 3, pp. 227-231, 2015.

[36] H. Sadeghi, S. Bailey, and C. J. Lambert, "Silicene-based DNA nucleobase sensing," Applied Physics Letters, vol. 104, no. 10, Article ID 103104, 2014.

[37] R. G. Amorim and R. H. Scheicher, "Silicene as a new potential DNA sequencing device," Nanotechnology, vol. 26, no. 15, Article ID 154002, 2015

[38] B. Aufray, A. Kara, Ś. Vizzini et al., "Graphene-like silicon nanoribbons on $\operatorname{Ag}(110)$ : a possible formation of silicene," Applied Physics Letters, vol. 96, no. 18, Article ID 183102, 2010.

[39] P. De Padova, C. Quaresima, C. Ottaviani et al., "Evidence of graphene-like electronic signature in silicene nanoribbons," Applied Physics Letters, vol. 96, no. 26, Article ID 261905, 2010.

[40] P. De Padova, O. Kubo, B. Olivieri et al., "Multilayer silicene nanoribbons," Nano Letters, vol. 12, no. 11, pp. 5500-5503, 2012.

[41] M. R. Tchalala, H. Enriquez, A. J. Mayne et al., "Formation of one-dimensional self-assembled silicon nanoribbons on $\mathrm{Au}(110)$ (2×1)," Applied Physics Letters, vol. 102, no. 8, Article ID 083107, 2013.

[42] G. Liu, Y. Wu, Y.-M. Lin et al., "Epitaxial graphene nanoribbon array fabrication using BCP-assisted nanolithography," ACS Nano, vol. 6, no. 8, pp. 6786-6792, 2012.

[43] J. G. Son, M. Son, K.-J. Moon et al., "Sub-10 nm graphene nanoribbon array field-effect transistors fabricated by block copolymer lithography," Advanced Materials, vol. 25, no. 34, pp. 4723-4728, 2013.

[44] S. M. Aghaei, N. Yasrebi, and B. Rashidian, "Characterization of line nanopatterns on positive photoresist produced by scanning near-field optical microscope," Journal of Nanomaterials, vol. 2015, Article ID 936876, 7 pages, 2015.

[45] P. Wagner, V. V. Ivanovskaya, M. Melle-Franco et al., "Stable hydrogenated graphene edge types: normal and reconstructed Klein edges," Physical Review B-Condensed Matter and Materials Physics, vol. 88, no. 9, Article ID 094106, 2013.

[46] Y.-L. Song, Y. Zhang, J.-M. Zhang, D.-B. Lu, and K.-W. Xu, "Modulation of the electronic and magnetic properties of the silicene nanoribbons by a single C chain," European Physical Journal B, vol. 79, no. 2, pp. 197-202, 2011.

[47] M. E. Dávila, A. Marele, P. De Padova et al., "Comparative structural and electronic studies of hydrogen interaction with isolated versus ordered silicon nanoribbons grown on $\mathrm{Ag}(110)$," Nanotechnology, vol. 23, no. 38, Article ID 385703, 2012.

[48] Y. Liang, V. Wang, H. Mizuseki, and Y. Kawazoe, "Band gap engineering of silicene zigzag nanoribbons with perpendicular electric fields: A Theoretical Study," Journal of Physics Condensed Matter, vol. 24, no. 45, Article ID 455302, 2012.

[49] C. Xu, G. Luo, Q. Liu et al., "Giant magnetoresistance in silicene nanoribbons," Nanoscale, vol. 4, no. 10, pp. 3111-3117, 2012.

[50] S. M. Aghaei and I. Calizo, "Band gap tuning of armchair silicene nanoribbons using periodic hexagonal holes," Journal of Applied Physics, vol. 118, no. 10, Article ID 104304, 2015. 
[51] H. Dong, D. Fang, B. Gong, Y. Zhang, E. Zhang, and S. Zhang, "Electronic and magnetic properties of zigzag silicene nanoribbons with Stone-Wales defects," Journal of Applied Physics, vol. 117, no. 6, Article ID 064307, 2015.

[52] Q. G. Jiang, J. F. Zhang, Z. M. Ao, and Y. P. Wu, "Density functional theory study on the electronic properties and stability of silicene/silicane nanoribbons," Journal of Materials Chemistry C, vol. 3, no. 16, pp. 3954-3959, 2015.

[53] S. M. Aghaei and I. Calizo, "Bandgap changes in armchair silicene nanoribbons perforated with periodic nanoholes," in Proceedings of the IEEE SoutheastCon (SECon '15), pp. 1-6, April 2015.

[54] S. M. Aghaei, M. M. Monshi, I. Torres, and I. Calizo, "Edge functionalization and doping effects on the stability, electronic and magnetic properties of silicene nanoribbons," RSC Advances, vol. 6, no. 21, pp. 17046-17058, 2016.

[55] S. M. Aghaei and I. Calizo, "Density functional theory study on energy band gap of armchair silicene nanoribbons with periodic nanoholes," MRS Advances, vol. 1, no. 22, pp. 1613-1618, 2016.

[56] M. Brandbyge, J.-L. Mozos, P. Ordejón, J. Taylor, and K. Stokbro, "Density-functional method for nonequilibrium electron transport," Physical Review B, vol. 65, no. 16, Article ID 165401, 2002.

[57] J. Taylor, H. Guo, and J. Wang, “Ab initio modeling of quantum transport properties of molecular electronic device," Physical Review B, vol. 63, no. 24, Article ID 245407, 2001.

[58] Atomistix Toolkit version 2015.0, QuantumWise, Copenhagen, Denmark, http://www.quantumwise.com.

[59] T. Wassmann, A. P. Seitsonen, A. M. Saitta, M. Lazzeri, and F. Mauri, "Structure, stability, edge states, and aromaticity of graphene ribbons," Physical Review Letters, vol. 101, no. 9, Article ID 096402, 4 pages, 2008.

[60] D. R. Stull and H. Prophet, JANAF Thermochemical Tables, No. NSRDS-NBS-37, National Standard Reference Data System, 1971. 

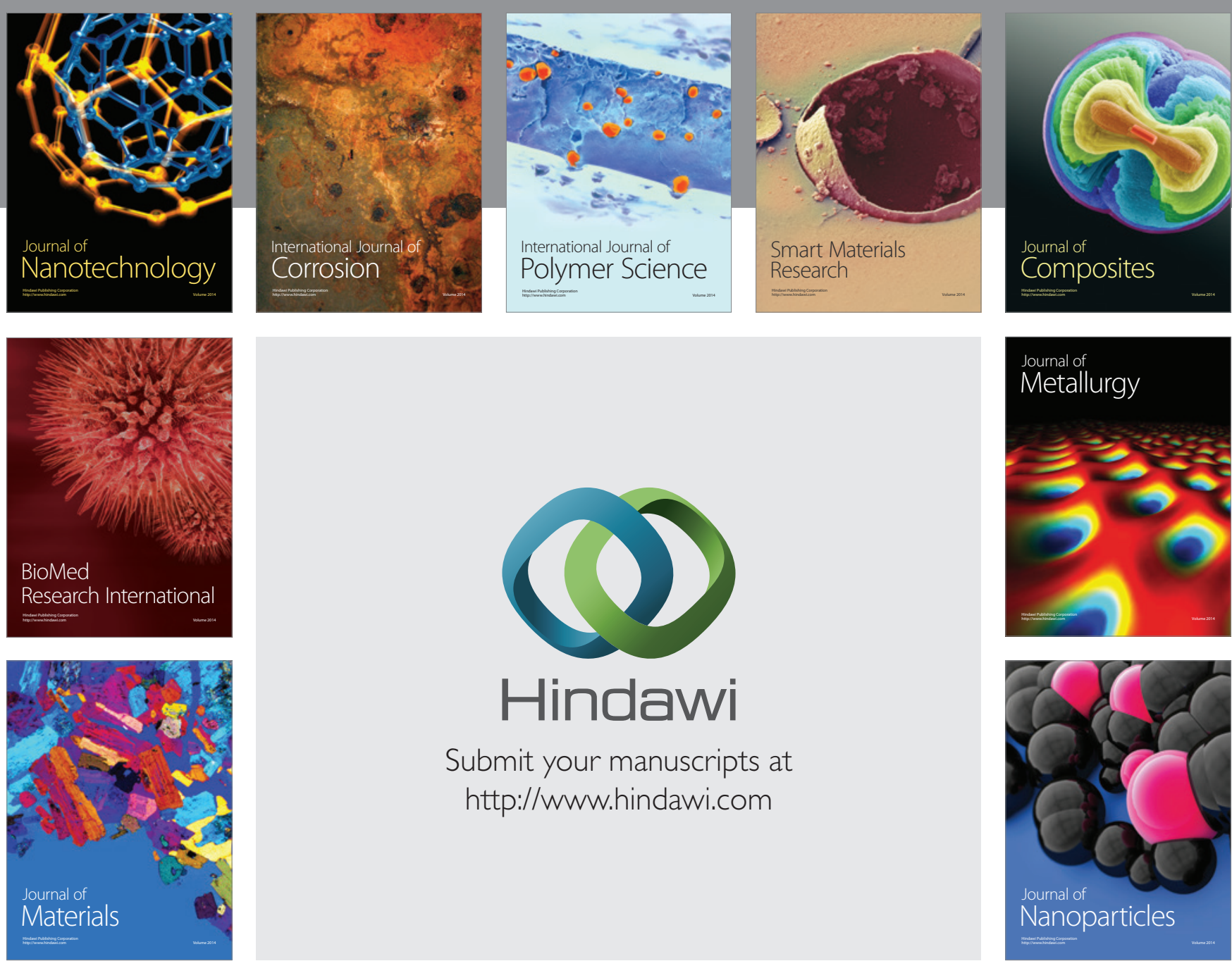

\section{Hindawi}

Submit your manuscripts at

http://www.hindawi.com

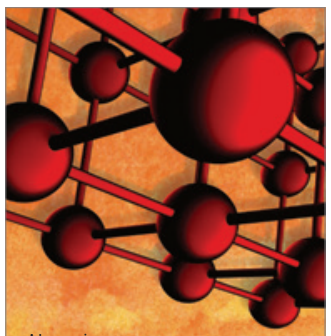

Materials Science and Engineering
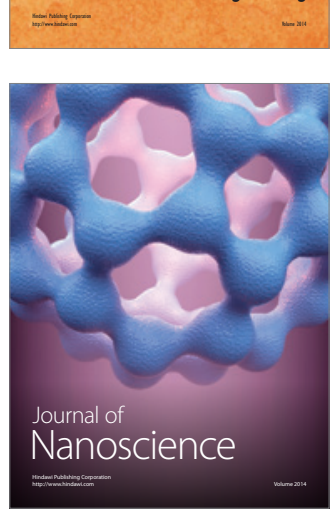
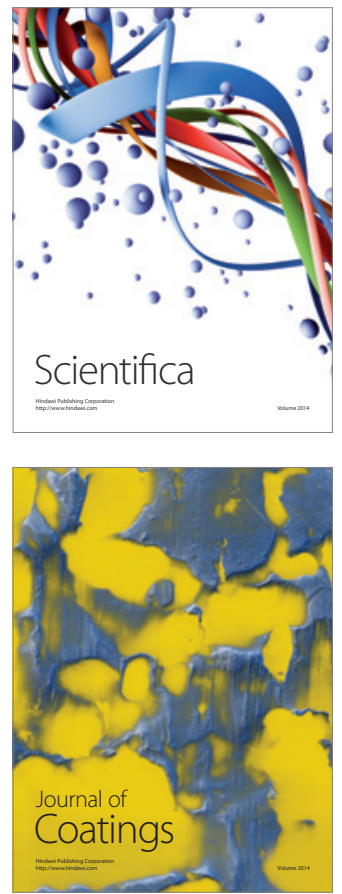
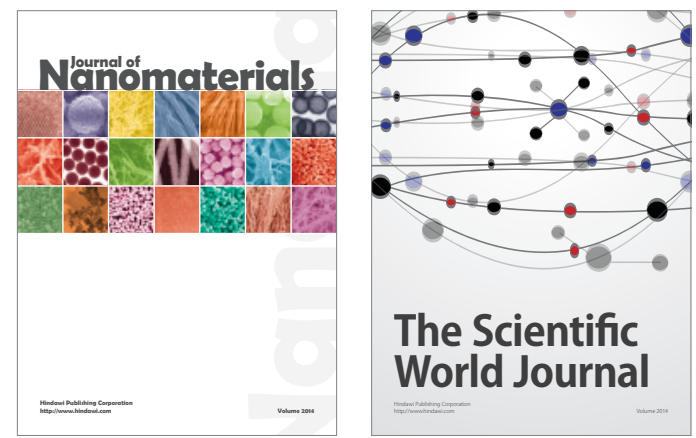

The Scientific World Journal
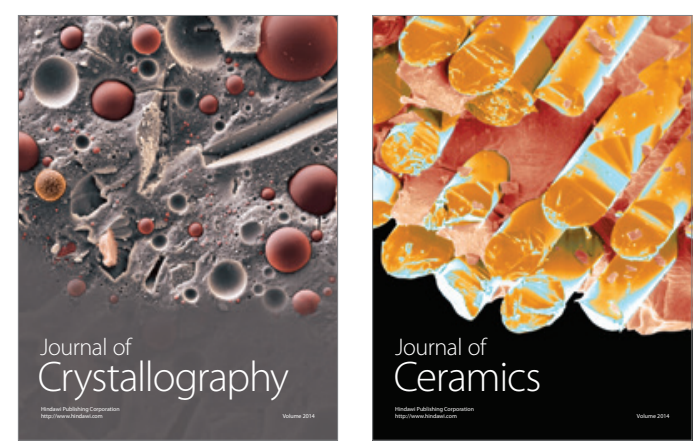
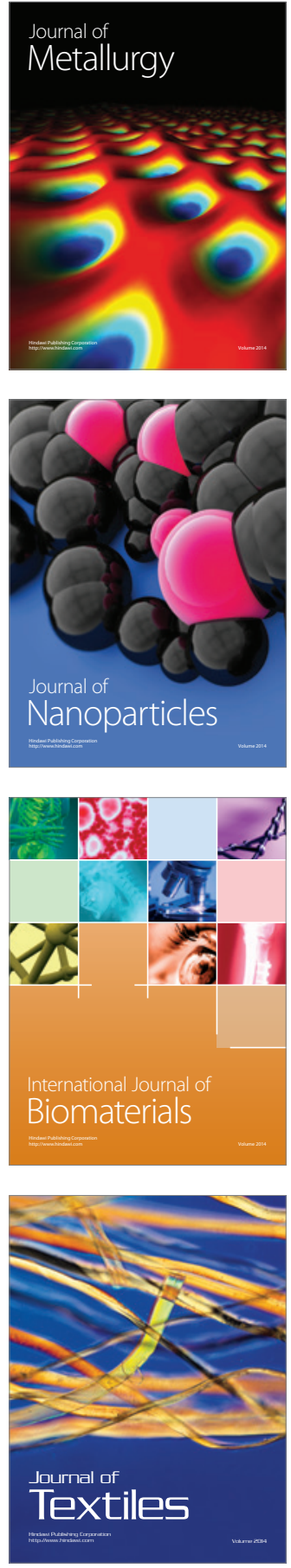